\title{
Percepciones de los ciudadanos catalanes acerca de la participación del público y los expertos en las controversias sobre el agua ${ }^{1}$
}

\author{
Juan C. Aceros \\ Francisco Tirado \\ Miquel Domènech
}

Universitat Autònoma de Barcelona. Departament de Psicologia Social.

Grup d'Estudis Socials de la Ciència i la Tecnologia (GESCIT)

juancarlos.aceros@uab.cat

franciscojavier.tirado@uab.cat

miquel.domenech@uab.cat

Recibido: 09-04-2008

Aceptado: 01-02-2010

\section{Resumen}

La gestión sostenible del agua ha aparecido en España como el centro de una controversia de primera magnitud. En ella, la participación ciudadana es un eje fundamental. A partir del análisis de grupos de discusión realizados en seis ciudades de Cataluña, este trabajo reflexiona sobre la percepción de la participación en los debates sobre el agua. Se presentan cinco actores que aparecen en el discurso de los entrevistados: los «expertos", los "perjudicados», los «beneficiados», los «interesados» y los «concienciados». Posteriormente, se examinan los criterios que permiten identificar, entre estos actores, los que son considerados legítimos en las controversias hidrológicas.

Palabras clave: percepción social; controversias tecnocientíficas; participación pública; políticas hidrológicas.

Abstract. Catalonian Citizens' Perceptions on Public and Expert Participation in Water Controversies

Sustainable water management has become the focal point of a highly important issue in Spain. Citizens' participation in this controversy it is fundamental. Having carried out the analysis of several focus groups in six Catalonian cities (Spain), this study reflects on the perception of participating in discussions on water. Five actors mentioned in interviewees' discourse are presented: the "experts», the "affected», the «benefited», the "interested», and the "conscious». Then, the criteria permitting to identify among these actors those considered to be legitimate participants in hydrological controversies are examined.

Key words: social perception; scientific and technological controversies; public participation; hydrological policies.

1. Este estudio ha sido financiado por el Ministerio de Educación y Ciencia a través del proyecto Ciencia, tecnología y ciudadanía en la sociedad del conocimiento (SEJ 2005-09319CO3-01/SOCI). 


\section{Sumario}

1. Controversias sobre el agua: contexto y cuestiones relativas a la participación

2. Diseño metodológico
3. Participación y conocimiento en las controversias sobre el agua

4. Conclusiones

5. Referencias bibliográficas

Este artículo responde a un interés por la creciente tecnificación de las controversias públicas en las llamadas «sociedades del conocimiento». Forma parte de un estudio sobre las implicaciones que tiene la participación de expertos y ciudadanos en la toma de decisiones relacionadas con asuntos tecnocientíficos que atañen a la sociedad en general. Tal cuestión se examina aquí en el marco de la gestión, el gobierno y la planificación del agua en España. En el primer apartado del artículo, se propone una introducción a este campo; allí se contextualizan brevemente los debates sobre el agua, resaltando la importancia que la participación pública ha venido adquiriendo en ellos. En el segundo apartado, se presenta la estrategia de investigación sobre la que se sustenta nuestro análisis. En el tercer apartado, se examina lo que los participantes en un conjunto de grupos de discusión expresan sobre los actores que intervienen en las controversias sobre el agua. Se pone especial acento en lo dicho acerca del papel de los expertos y diferentes clases de ciudadanos que aparecen en el discurso. De cada uno de ellos, se resalta el tipo de conocimiento que poseen y la posición que se les atribuye en los debates. Se concluye con una breve síntesis de lo expuesto y con algunas consideraciones referidas tanto a la importancia del conocimiento experto en las controversias, como a la necesidad de examinar la «identidad territorial» como un fenómeno psicosocial que parece intervenir en la comprensión de la participación en las políticas y los planes hidrológicos.

\section{Controversias sobre el agua: contexto y cuestiones relativas a la participación}

El interés por desarrollar sistemas que permitan una correcta planificación, gobierno y gestión de los recursos hídricos se ha convertido en un asunto de primer orden a partir de la mitad del siglo XX. Desde la redacción de la Carta Europea del Agua (Estrasburgo, 1968), la Conferencia del Agua de las Naciones Unidas (Mar del Plata, 1977) y, particularmente, desde la Declaración de Río sobre Ambiente y Desarrollo (1992), la gestión eficiente y sostenible de dichos sistemas, ya sea en relación con la agricultura de irrigación, con la producción hidroeléctrica o con la provisión de servicios de agua y saneamiento, es una de las cuestiones de mayor importancia en el mundo.

En el caso del Estado español, el agua ha aparecido en los últimos años como «un nuevo tema ambiental en la política nacional» (Jiménez, 2005: 118). 
Desde la Ley 29/1985 de aguas, se ha hecho obligatorio elaborar un plan hidrológico nacional que coordine los proyectos emprendidos a un nivel de cuenca. El proceso de planificación consecuente ha activado una amplia oposición social (Arrojo, 2001). En respuesta a una prolongada historia de políticas hidrológicas orientadas a la construcción de grandes infraestructuras — subvencionadas por el Estado con la intención de favorecer el regadío y la producción hidroeléctrica (Arrojo, 2006; Tàbara et al., 2004)—, desde la década de 1980, empiezan a visibilizarse discursos críticos que proponen nuevas formas de enfocar la gestión del agua. En la década de 1990, las movilizaciones contra el Plan Hidrológico Nacional reúnen a diferentes actores que denuncian sus efectos perjudiciales sobre el potencial de desarrollo de diferentes regiones de España (Boné, 2003), su insostenibilidad económica (Albiac et al., 2003), su falta de apoyo científico (Arrojo, 2001) y su desatención a las últimas tecnologías y formas de gestión del agua (Embid, 2003).

A diferencia de las controversias sobre el agua que se habían desarrollado con anterioridad, donde fue dominante el debate entre expertos y políticos (Mülberger et al., 2007), en la oposición al Plan Hidrológico Nacional intervinieron activamente ciudadanos interesados o afectados, muchos de los cuales integraban grupos ecologistas y movimientos territoriales (Tàbara et al., 2004). La creación de espacios abiertos a la participación de estos actores ha estado acompañada de tres fenómenos. En primer lugar, la capacidad del movimiento ecologista español para formular la política ambiental a partir de la generación de conflicto a fin de crear impacto (Jiménez, 2005). En segunda instancia, la prescripción de la consulta y la implicación pública en el derecho comunitario - particularmente de la Directiva Marco del Agua (Álvarez, 2006; La Calle, 2006). En último lugar, la ya conocida insatisfacción frente a los sistemas de discusión, evaluación y toma de decisiones sobre asuntos tecnocientíficos que sólo tienen en cuenta el criterio experto (Einsiedel et al., 2001) y la revisión el papel de los científicos, los gobiernos y el público en la regulación de los procesos de toma de decisiones (Aibar, 2002b, Luján y Todt, 2007).

Como consecuencia de lo anteriormente mencionado, cada vez es más difícil que un asunto de interés público, como la gestión del agua, se sustraiga del debate abierto, porque se le considera un «asunto técnico» (Graña, 2005). Los pronunciamientos acerca de la seguridad, la viabilidad o la utilidad de proyectos concretos están cada vez más sometidos al escrutinio y al cuestionamiento de los medios, las organizaciones no gubernamentales, las entidades gubernamentales y los sectores comprometidos de la sociedad civil (Stilgoe, 2007). De allí el uso cada vez más extendido de mecanismos consultivos y de participación directa, como las conferencias de consenso, las audiencias públicas, los paneles ciudadanos, los grupos temáticos, la gestión negociada y el referéndum (Aibar, 2002b; Goven, 2003; Lezaun y Soneryd, 2007). El adecuado diseño y ejecución de estos mecanismos requiere del examen de lo que la participación ciudadana es e implica. Comprender tales cuestiones, desde la óptica de los sujetos llamados a integrarse en las contro- 
versias tecnocientíficas, puede ayudar en la democratización de las políticas ambientales en general, y de las hidrológicas en particular. En coherencia con estas afirmaciones, en el presente artículo examinamos cómo la participación ciudadana en las controversias hidrológicas es percibida socialmente en Cataluña (España). Hemos ubicado nuestro estudio en esta comunidad autónoma porque el debate en torno a las políticas hidrológicas nacionales ha sido particularmente intenso, además de ser ampliamente conocido por la población en general. Para realizar el análisis, utilizamos extractos de una serie de grupos de discusión llevados a cabo en diferentes poblaciones del territorio catalán durante el año 2006.

\section{Diseño metodológico}

Este artículo se deriva de una investigación más amplia llevada a cabo mediante once grupos de discusión en los que se ha debatido el tema objeto de nuestra atención. En total, se efectuaron tres en Barcelona, dos en Girona, uno en Granollers, dos en Tarragona, uno en Lleida, uno en Terrassa y uno en Amposta. Se cuidó que los grupos estuvieran equilibrados en cuando al género y que representaran diferentes rangos de edad (jóvenes, adultos y adultos mayores). Lo que hacía semejantes a los participantes era su procedencia, por lo que fueron incluidos en los grupos de discusión en virtud de su lugar de residencia habitual. Atendiendo a tal criterio, también se contó con la presencia (minoritaria) de extranjeros y de personas que han participado en actividades y/o colectivos relacionados directamente con las controversias sobre el agua. De esta manera, también se esperaba aumentar la heterogeneidad de la muestra, evitar que el discurso fuera redundante (Rubio y Varas, 1997) y facilitar el surgimiento del debate.

Para iniciar los grupos de discusión, se presentaban dos anuncios de periódico que manifestaban posiciones distintas respecto al trasvase del Ebro. A partir de las primeras intervenciones, la discusión fluía de manera espontánea. Con el fin de orientarla hacia los objetivos de la investigación, el moderador manifestaba su interés por conocer la opinión de los asistentes sobre (1) el papel de los expertos, los políticos y los ciudadanos en la controversia, (2) las distintas formas de participación de la sociedad civil en el asunto, (3) los medios a través de los cuales las personas obtienen conocimiento e información sobre el debate y sus detalles científico-técnicos. El análisis posterior del material estaba orientado a identificar la estructura de lo dicho por los participantes (Rubio y Varas, 1997). Para lograrlo, se contó con un conjunto de categorías deductivas que se correspondían con los temas antes mencionados. Adicionalmente, se añadieron otras categorías referentes a temas que aparecieron en las diferentes sesiones.

Durante el desarrollo de los grupos de discusión, se hizo patente que las controversias hidrológicas no son ajenas a los ciudadanos catalanes y que éstos coinciden en considerarlas una cuestión relevante en la sociedad actual. A partir de tal reconocimiento, los asistentes defienden que participar en los deba- 
tes acerca del agua forma parte de sus derechos y deberes. Sin embargo, también sostienen que, para intervenir, requieren de un conjunto de condiciones, entre las que sobresale la posesión de ciertos tipos de conocimiento. A continuación, analizamos lo expresado al respecto, acompañando nuestras interpretaciones con formulaciones de los propios entrevistados.

\section{Participación y conocimiento en las controversias sobre el agua}

\subsection{Saber ciencia para participar}

Un tema obligado durante los debates entre los entrevistados atañe a la posesión, por parte de los ciudadanos, de conocimiento relevante sobre las controversias hidrológicas. Este conocimiento es, en principio, información científico-técnica usada para tomar decisiones y justificar la orientación de las políticas ambientales. Participar en una controversia sobre el agua requiere conocer cuestiones como el estado de los ríos, su biodiversidad, la dimensión de los caudales, las fuentes posibles de contaminación, la viabilidad de la desalación, etc. Sin embargo, no son pocos entre los entrevistados que aseguran no estar suficientemente informados sobre dichos tópicos:

Yo me siento muy desinformada. (N4.Girona1.30)

[...] de la situación del agua, tampoco soy demasiado entendido. (H4.Granollers.9)

Yo tampoco tengo una opinión tan elaborada. (H3.Tarragona1.12)

Expresiones como las anteriores no implican un desconocimiento absoluto. En las dos últimas, se encuentran matizaciones que nos hacen pensar en la existencia de un saber mínimo. Afirmar que no se es «demasiado entendido» o que no se tiene "una opinión tan elaborada» supone la posesión de algún grado de información que, sin embargo, es valorada como insuficiente por los entrevistados. Así, los conocimientos que ellos mismos manifiestan durante los grupos de discusión son autoevaluados como carentes de la profundidad que requieren los procesos consultivos y decisorios:

Lo que pasa, por ejemplo, si me dijeran que tengo que decidir realmente, se me haría muy difícil, porque yo no estoy suficientemente informada, no me vería capaz de hacerlo, ¿no? O sea, por lo tanto, si a mí me dijeran «decídelo», yo creo que me abstendría. (D1.Barcelona3.22)

En este fragmento, se aprecia el carácter «insuficiente» del conocimiento que los asistentes se atribuyen a sí mismos. Se encuentra también la utilidad de esta autovaloración al justificar la ausencia de implicación en procesos de planificación, gobierno y gestión de los asuntos hídricos. Dichos fenómenos podrían interpretarse como una prueba de que los ciudadanos requieren formación científico-técnica para adquirir un papel relevante en 
las controversias ${ }^{2}$ o de que, simplemente, deben ser suplantados por expertos en temas ambientales. En efecto, algunos entrevistados señalan la necesidad de aumentar la educación de la población en materia hidrológica o de dejar la definición de las políticas en manos de científicos y técnicos. Sin embargo, estas ideas no son las que más se manifiestan en los grupos de discusión. Frecuentemente, el conocimiento experto no es simplemente solicitado, sino más bien reposicionado. $\mathrm{Al}$ respecto, obsérvense las siguientes intervenciones:

Yo creo que se tienen que hacer informes científicos, se tiene que informar de todas las variables, cuáles son las posibilidades que se tienen para solucionar determinados problemas. Pero yo creo que, al final, el que vive en el río tiene que decir: «Si a mi no me importa, si es esto el problema, darte tanto»; y el que la tiene que recibir [el agua] decir... «Bueno, si no es del río la cojo de otro sitio, de desalinización» o de lo que sea. (N2.Barcelona1.10)

Tampoco estoy de acuerdo que un experto tenga más derecho a opinar que uno que vive allí. Es decir, si yo vivo allí tendría más derecho por vivir allí, porque un experto hace sus planes, sus teorías científicas, pues muy bien ¿no? Es su opinión, pero el que vive allí simplemente porque siempre ha vivido allí y le gusta ver el río lleno de agua me parece un derecho. (H2.Barcelona2.6)

Como puede notarse, los entrevistados no rechazan la participación de los expertos en las controversias. Los especialistas tienen un rol determinado en ellas (por ejemplo: «hacer informes»), pero dicho papel no es más importante que el que pueden desempeñar otros actores. Su conocimiento ha de entenderse como una "opinión» equiparable a otras, y el «derecho» a expresarlo, como algo que no es privilegio exclusivo suyo. Así pues, los expertos pueden verse como sujetos en relación con otros y cuyo saber ha de entrar en negociación con el conocimiento de esos otros. En concreto, se hace referencia a las personas directamente afectadas por los proyectos hidrológicos («[...] el que vive allí simplemente porque siempre ha vivido allí [...]»).

La reconsideración del papel de los expertos está asociada con la valoración positiva de la "opinión» del lego. Este último, en virtud de su experiencia, deviene interlocutor válido en las controversias hidrológicas. Adviértase que, en los fragmentos que examinamos, los entrevistados no recurren al discurso de la «información insuficiente». Los actores locales son vistos como participantes de pleno derecho en virtud de sus conocimientos. Son lo que puede llamarse «expertos

2. Dentro de los estudios sobre la comprensión pública de la ciencia, este argumento se conoce como «modelo del déficit». Se refiere a la idea según la cual el público se resiste a la ciencia y a la tecnología porque no comprende suficientemente la información especializada (Aibar, 2002a; Stenekes et al., 2006) y sostiene que la falta de alfabetización tecnológica estaría en la base de la ineficiencia de los ciudadanos a la hora de juzgar asuntos científicotécnicos de interés público y de participar en la definición de políticas científicas (Tytler et al., 2001; Sturgis y Allum, 2004). 
contributivos no certificados» (Aibar, 2002b) ${ }^{3}$. Dichos legos expertos amplían lo que puede considerarse conocimiento legítimo en una controversia. La autoridad de su saber no viene marcada por un aval institucional — como en el saber especializado-, sino por la experiencia cotidiana y por los efectos que tiene en sus vidas la toma de decisiones acerca de la controversia. El siguiente fragmento también puede ser interpretado en esta dirección:

[Los expertos] tampoco tienen que tener el derecho final de todo por saber un par de libros más, ¿̇no? Bien, su opinión, bien, muy bien, escuchar de todo; pero al final, yo creo más a un campesino de esa zona que a un biólogo que ha leído libros, porque al final..., por tradición, por cultura, por vivir, porque sus padres vivían así..., sabe mejor sus intereses. (H2.Barcelona2.9)

Afirmaciones como estas recuerdan el estudio clásico de Collins y Pinch (2002) sobre controversias en las que se resalta cómo los granjeros del Reino Unido, afectados por la catástrofe de Chernobil, perdían autonomía bajo la jurisdicción de una lejana burocracia científica, y cómo su saber local derivado del conocimiento de las condiciones del terreno podía ofrecer datos relevantes sobre las radiaciones y su medición. El fragmento también remarca que el conocimiento de "un campesino de esa zona» no se refiere solamente a su entorno natural, sino también a su posicionamiento político-económico («sabe mejor sus intereses»). Así, apuntala el valor del saber — tanto «natural» como «social»— del lego, pero hace algo más: traduce la forma de concebir las controversias tecnocientíficas. Éstas no aparecen como debates sobre «hechos» o «pruebas», sino como una "lucha de poderes» (D1.Barcelona3.62).

La traducción de las controversias tecnocientíficas en «luchas de intereses» o «luchas de poder» reposiciona a los actores que se ven comprometidos en los debates hidrológicos. Las personas afectadas no son solamente «expertos contributivos no certificados», sino actores que defienden su propio provecho. Los científicos y técnicos, por su parte, dejan de ser quienes aportan el argumento neutral y definitivo que cierra los debates, para convertirse también en garantes de intereses concretos. Dicha posición es, en cierto sentido, problemática para estos últimos actores. El conocimiento que detentan, tradicionalmente visto como objetivo e imparcial, pierde dicho estatus para apreciarse como ligado a fenómenos que no son estrictamente científico-técnicos:

Yo pienso que sí y seguramente están basados y tienen una base científica muy correcta y muy adecuada. Lo que interese que salga a la luz o que no salga a la luz es otro tema... que puede interesar a unos o a otros. «Esto lo escondemos porque esto no..., ahora no interesa que salga», 0 «lo sacamos en el momento mas oportuno». (D1.Tarragona1.11)

3. Los expertos contributivos no certificados son determinados grupos del público no científico que - en virtud de su experiencia continuada sobre las circunstancias locales en las que desarrollan sus actividades - poseen conocimientos relevantes para la controversia (Aibar, 2002b). 
[... ya he vivido unos años en este mundo para darme cuenta de por qué hacen estas cosas, ¿no? Que no las hacen porque les interesa la naturaleza, ni porque quieren dar de beber a las personas..., ¿no? Las hacen porque tienen amigos en tal constructora [...] Muchas veces, son expertos que han estudiado muchísimo, pero luego no tienen interés para ir al centro de la cuestión. (H2.Barcelona2.10)

Los anteriores extractos ponen en boca de los ciudadanos lo que Espluga (2004) ha resaltado a propósito de la gestión de riesgos tecnológicos: que las estimaciones e interpretaciones de los expertos no son axiológicamente independientes y que suponen una perspectiva política o ideológica. Este reconocimiento ha sido rastreado por Lujan y Todt (2007) en su estudio sobre la percepción del «principio de precaución» en España. Según estos últimos autores, los ciudadanos españoles, por un amplio margen, consideran que los científicos pueden estar influidos por valores e intereses económicos. Así lo dan a entender también los entrevistados en nuestro estudio cuando resaltan que las investigaciones, y su uso, pueden transformarse debido a las coyunturas políticas, las orientaciones de las entidades que financian a los especialistas, el posicionamiento político de los expertos, la influencia de grupos de presión o la corrupción política.

En los fragmentos, también se percibe una disminución del valor del saber especializado. Así pues, en los grupos de discusión, la participación de los expertos no solo es equiparada a la de otros actores, sino que se le resta importancia e, incluso, se le deslegitima. Los entrevistados manifiestan darle más crédito al "perjudicado» ("Yo creo más a un campesino de esa zona», H2.Barcelona2.9). Desconfían de la relación percibida entre el conocimiento experto y las ideologías políticas o los intereses económicos, porque les impide «ir al centro de la cuestión", es decir, participar de manera determinante (y ecuánime) en los procesos de toma de decisión política.

A medida que el saber especializado pierde su estatus de objetividad, y que el saber del lego ve aumentada su autoridad, se abren oportunidades para la participación ciudadana. Esta última aparece como necesaria en un contexto conflictivo en el que se reivindican los intereses de los "perjudicados» y se procura salvaguardarlos frente a los de otros colectivos que desconocen (o pretenden desconocer) los efectos negativos de los planes hidrológicos sobre ciertas poblaciones. Al examinar las condiciones en las que hacen deseable esta participación, los entrevistados negocian sobre el significado del conocimiento experto en las controversias sobre el agua. Lo hacen discutiendo sobre la forma como es asimilado, utilizado o cuestionado por actores distintos a los científicos y los técnicos. Como resultado de este proceso, junto a los especialistas y los «perjudicados», aparecen en el discurso otros actores que, aunque pueden integrarse en los debates sobre el agua, tienen un estatus epistemológico y una legitimidad percibida diferente en cada caso.

\subsection{Clasificación y cualificación de los ciudadanos en virtud de su saber}

Hasta este momento hemos mostrado cómo, en el discurso de los entrevistados, el saber experto no simplemente es reclamado, sino también reposicio- 
nado. Al hacerlo, la información científico-técnica ve disminuido su valor, deja de percibirse como el requisito sin el cual la participación en debates hidrológicos es imposible, y se convierte en una "opinión» que puede ser contrastada con otras. De esta manera, los ciudadanos pueden situarse como interlocutores válidos en las controversias hidrológicas, en la medida que sus propios valores y juicios pueden ser reconocidos.

Llegados a este punto, conviene preguntarse: «iDe qué ciudadanos estamos hablando?». Como ya hemos indicado, el discurso apunta a que las personas que viven cerca de los ríos objeto de debate han de ser las participantes legítimas en las controversias sobre el agua. Son ellas las que ven aumentada la autoridad de su saber y las que ven reconocidos sus intereses locales. Sin embargo, en los grupos de discusión también son mencionados otros ciudadanos a quienes se les asignan, en virtud de su saber, distintas posiciones en los debates hidrológicos. Para referirse a ellos, los entrevistados usan las categorías de «beneficiados», "concienciados» 0 «interesados».

Durante los grupos de discusión, la palabra beneficiados suele aplicarse a los usuarios domésticos que tienen asegurado su consumo diario gracias a los planes hidrológicos ${ }^{4}$. Algunos entrevistados consideran que dichos actores deberían ser participantes regulares de los debates sobre el agua:

[...] yo pienso que se debe dar voz a los usuarios del recurso, ¿no? Que si se habla de un trasvase [...] pues la gente beneficiaria o perjudicada por las consecuencias de este trasvase pues debe sentirse representada, debería dar su punto de vista. (H2.Girona.20)

En este fragmento, la posible implicación de la «gente beneficiaria» es puesta en relación de igualdad con la participación de la "gente perjudicada» (es decir, de los actores locales). Los dos tipos de ciudadanos pueden intervenir en las controversias hidrológicas, ya sea mediante otros actores que actúan en su nombre o expresando sus preocupaciones directamente («debe sentirse representada, debería dar su punto de vista»). Sin embargo, en el desarrollo de los grupos de discusión, resulta habitual que esta simetría sea cuestionada.

Ya hemos mencionado, en páginas anteriores, que los «perjudicados» son vistos como agentes que poseen un conocimiento válido que les autoriza a participar. Ahora es necesario resaltar que, por el contrario, los "beneficiados» suelen ser percibidos como sujetos carentes de conocimiento seguro. Así, al entrevistado antes citado le responde otro en los siguientes términos:

4. También se suele denominar «beneficiados» a miembros de diferentes sectores económicos que obtienen ganancias de la planificación hidrológica; sin embargo, aquí reservamos para ellos el término «interesados». Obramos así, no solo porque los entrevistados lo hacen con frecuencia, sino porque de esta manera se resalta el carácter activo de los actores económicos en la controversia. De los «interesados», se afirma (o se sugiere) que realizan acciones a favor de sus intereses; mientras que los «beneficiados» simplemente obtienen provecho de lo gestionado por otros. 
[...] estoy totalmente de acuerdo con eso que dices; pero yo pienso que hay otro punto que es, que para que estos ciudadanos puedan hacer y puedan dar su opinión, deberían partir de una información totalmente imparcial. (N1.Girona1.21)

En esa intervención, no se descarta que los «beneficiarios» puedan tener algo que aportar a las controversias sobre el agua. Sin embargo, se sugiere que cuentan con información incompleta (o, más bien, sesgada) respecto a los temas sobre los que se discute. Al igual que los expertos y los "perjudicados», solo tienen un conocimiento parcial; sin embargo, en el primer caso, este conocimiento está sustentado científicamente y, en el segundo, experiencialmente. Los conocimientos de especialistas «seguramente [...] tienen una base científica muy correcta» (D1.Tarragona1.11); la persona que vive junto a un río que va a ser trasvasado, "por tradición, por cultura, por vivir, porque sus padres vivían así..., sabe mejor sus intereses» (H2.Barcelona2.22). El conocimiento de los «beneficiados», por su parte, está prefigurado por otros. Su opinión y voluntad no le pertenecen, son los que un segundo tipo de actor le implanta:

Yo no veo que el mundo científico tenga una plataforma de difusión que haga llegar realmente aquello que esta estudiando de una manera clara [...] Hay revistas científicas que entre ellos lo saben todo; pero a nosotros no nos llega eso. [...] Si la ciudadanía nos tenemos que fiar de los medios de comunicación, yo ya hace mucho tiempo que dije que se había terminado, que no confiaba en ellos. Que veo que hay unos intereses detrás muy grandes y que, a veces, no tenía que ver con los partidos políticos, sino que tiene más que ver con la economía y los intereses económicos. (H2.Terrassa.12)

El conocimiento parcial de los "beneficiados", mediatizado como es, se ve deslegitimado por la falta de transparencia de las fuentes de las que es obtenido. Estos actores no podrían expresar un juicio adecuado sobre las cuestiones hídricas, debido a que están influidos por la propaganda emitida a través de los medios de comunicación. En tal dirección, los entrevistados resaltan la existencia de «intereses detrás muy grandes» que determinan el tipo de conocimiento que llega a los ciudadanos. Así, los medios, como los expertos, pueden aparecer como herramientas empleadas por actores «interesados» en que las controversias sobre el agua se clausuren a favor de su propio beneficio.

La categoría «interesados» suele reunir a dos tipos de actores: (1) los partidos políticos y las administraciones, que buscan mantener una tendencia de voto favorable, prometiendo y/o llevando a cabo determinadas intervenciones hidráulicas $^{5}$, y (2) los representantes de diferentes sectores económicos (la agricultura, el turismo, la construcción, la producción hidroeléctrica, etc.), que pueden obtener ganancias con dichos proyectos. Estos agentes "interesados»,

5. Estos actores también pueden estar interesados en obtener ganancias económicas; en cuyo caso los entrevistados hacen referencia, fundamentalmente, a casos de corrupción política relacionada con el sector inmobiliario. 
independientemente o en connivencia, suelen aparecer en el discurso como poseedores de información privilegiada que solo entregan en la medida y forma que favorezcan a sus intereses. Su participación en la controversia pasar por el empleo estratégico del saber entregado por los expertos para justificar sus propuestas, así como por una difusión tendenciosa de dicho saber para orientar la opinión pública a su favor.

La forma de percibir a los «interesados» es especialmente llamativa, ya que algunos de los actores económicos que se mencionan (en concreto, los «regantes» y las "hidroeléctricas») son usuarios con título concesional que integran los órganos de gobierno de las confederaciones hidrográficas. Los mismos, hasta la década de 1990, han sido los únicos ciudadanos con posibilidad de participar en la gestión de cuencas. Por otro lado, los actores políticos (especialmente las administraciones), han servido habitualmente de mediadores entre diferentes intereses en disputa y han tomado las decisiones tendientes a regular, entre otras cosas, los asuntos medioambientales. La desconfianza que se expresa respecto a su participación en las controversias y al conocimiento que ofrecen (o que permiten ofrecer), puede ser interpretada como una manifestación de un descontento social frente a la forma como se han gestionado los temas hidrológicos en España hasta hace pocos años.

En este contexto de desconfianza — donde también puede incluirse la disminución de la relevancia que se le da a la «opinión» de los expertos y a la caracterización de la "opinión» del «beneficiado» como puramente ideológica (entendiendo ideología como sinónimo de mala conciencia)—, surge otro tipo de ciudadano que se percibe como participante legítimo en los debates hidrológicos. Ellos son los llamados «concienciados», definidos en el grupo de discusión de Tarragona como:

[...] la gente que busca [...] que entra en Internet. Hay gente que ya tiene conciencia porque ya se preocupa sobre el tema. (D1.Tarragona1.27)

Tales ciudadanos se encuentra también interesados por el desarrollo de las controversias, pero no porque puedan obtener beneficios económicos o políticos. En lo dicho por los entrevistados, los «concienciados» aparecen como simplemente atraidos o preocupados por los debates sobre el agua debido a que los consideran actuales e importantes. Son, de hecho, sujetos que buscan información por su cuenta y para satisfacer su propia curiosidad.

La identificación de estos actores establece una nueva clasificación. Así como existe, entre los ciudadanos, "gente perjudicada» y "gente beneficiada», también es posible encontrar "concienciados» $\mathrm{y}$ «no concienciados»:

Puede ser que la manera de cribar entre el concienciado y el no concienciado sería ceder más fácilmente toda la información sobre este tema. Poder alegar, poder moverte si estás interesado; pero no necesariamente que te vengan a consultar a casa sobre el tema que quieres hacer, ni que te bombardeen con información sesgada [...] Es decir, que quien quiera preocuparse por el tema 
pueda acceder fácilmente a la información, pueda formarse una opinión y pueda participar en el proceso de decisión. (H1.Girona2.9)

Del fragmento, puede derivarse que los «no concienciados» están exentos de preocupación por los temas hídricos y, en esta medida, no realizan acciones tendentes a obtener información sobre ellos. Se encuentran satisfechos con lo que se les entrega a través de lo que, en el fragmento, se denomina un «bombardeo de información sesgada». Son, de este modo, epistemológicamente idénticos a los «beneficiarios»: sujetos heterónomos carentes de conocimiento seguro para participar en las controversias. Como es de esperar, con frecuencia, los «beneficiados» son considerados, al mismo tiempo, ciudadanos «no concienciados»:

Aquí no vemos la [televisión] autonómica valenciana, pero seguro que durante todo el conflicto les estuvo diciendo «nos hace falta tanta agua, a Valencia nos hace falta tanta agua y estos desgraciados la están tirando». O sea, imagínate lo que les deberían llegar a decir, esta gente [...] Por eso te lo digo [el actor que debe tomar las decisiones]: no puede ser nadie de aquí ni de allí, tiene que ser alguien de fuera. (H1.Barcelona3.16)

En este fragmento, se puede advertir la necesidad sentida por algunos de los entrevistados de contar con agentes imparciales que medien entre los diferentes participantes de la controversia. La propuesta es coherente con la línea argumental que hemos venido siguiendo. Debido a que quienes intervienen en la controversia, sean "perjudicados» (los "de aquí») o «beneficiados» (los "de allí»), tienen intereses en juego, y que las autoridades políticas son puestas en duda como mediadores transparentes, el entrevistado sugiere la intervención de «alguien de fuera». En este caso, se refiere a una comisión de expertos de la Unión Europea ${ }^{6}$; sin embargo, los ciudadanos "concienciados» gozan de un estatus similar al de este mediador externo. De ellos se dice que pueden "participar en el proceso de decisión»(H1.Girona2.9), debido a que se han formado una opinión fundamentada a partir de información que ellos han consultado, contrastado y revisado críticamente:

Yo creo que eso, que si la información que te llega es de diferentes personas, de técnicos de diferentes partes, puedes agarrar más un criterio después [...]

6. El entrevistado lo expresa así: «se deberían buscar criterios objetivos, científicos, no sé. Una comisión europea que [...] evalúe este tipo de obras» (H1.Barcelona3.11). A la capacidad de ofrecer una mirada imparcial, otros entrevistados le suman una ventaja más a la participación de la "comisión europea»: su capacidad de frenar proyectos y planes hidrológicos controvertidos, debido a que las infraestructuras sobre las que se debate pueden estar financiadas con el Fondo de Cohesión de la UE («Porque mucha, o sea, mucha construcción del País Valenciano está pagada con fondos europeos. Entonces, también no es un problema tampoco de España; también es un poco de Europa, que permite hacer eso" (D4.Girona2.35)). 
También es eso, si tu tienes un criterio, y eso más en las generaciones más pequeñas, puedes educar desde la base a los pequeños y ya aprenderán eso: que tengan un criterio, que puedan opinar. (N1.Girona1.668)

Los "concienciados» pueden asegurarse una posición a partir de la revisión de fuentes diversas de información. Fuentes que, presumiblemente, incluirán tanto posturas a favor como en contra de planes o proyectos hidrológicos sometidos a debate. De allí que su posición pueda ser percibida como imparcial o, por lo menos, crítica. Su perspectiva es ciertamente crítica en la medida en que la «opinión» que detentan estos ciudadanos no ha sido simplemente asimilada, sino activamente construida: es un "criterio", un juicio, no una imposición. Su intervención también puede verse como imparcial, puesto que se elabora y se expresa en ausencia de relaciones directas con los beneficios o los perjuicios de un plan o proyecto hidrológico. El «concienciado» es visto como alguien que no tiene nada en juego, salvo su propio sentido moral.

Así pues, igual que una "comisión europea», el «concienciado» es una instancia potencialmente crítica e imparcial. Adicionalmente, puede interpretarse como la intervención de «alguien de fuera» que no solo estaría desvinculado de los intereses en disputa, sino que también se expresaría desde la distancia. Su aparición en el discurso introduce la posibilidad de que la participación ciudadana no se limite exclusivamente al ámbito de lo local. De esta manera, permite concebir a las controversias sobre el agua como espacios conflictivos donde, si bien debe reconocerse un lugar preponderante a los "afectados", puede participar cualquier persona legitimamente preocupada por los planes o los proyectos hidrológicos.

Los ciudadanos «concienciados», junto con los «expertos», los «afectados», los «beneficiados» y los «interesados», configuran la constelación de actores que, desde el punto de vista de los entrevistados, se da cita en las controversias sobre el agua. Su aparición en el discurso indica que los debates sobre temas de alto componente tecnocientífico, pero de gran relevancia pública, requieren de algo más que la emisión de un dictamen fundamentado en el saber especializado. Los planes y proyectos hidrológicos, como ha demostrado el amplio movimiento en contra del Plan Hidrológico Nacional de 2001 y como establece la Directiva Marco de Agua, ya no pueden sustraerse del examen público. Así lo indican también los grupos de discusión realizados, en los que ciertos tipos de ciudadanos son valorados como participantes legítimos que han de ser tomados en cuenta en los procesos consultivos y decisorios. Desatender a este hecho significa ir en contra de la percepción pública que ya no entiende las políticas y los planes hidrológicos concebidos, implementados y evaluados sin el concurso de las personas «afectadas» $\mathrm{y}$ «concienciadas».

\section{Conclusiones}

En este artículo, proponemos una mirada sobre las controversias tecnocientíficas públicas en España. Nos ha interesado particularmente examinar, desde 
la perspectiva de los ciudadanos catalanes, cómo se entiende la participación de expertos y del público en los debates en torno a intervenciones hidráulicas y a planes hidrológicos. Esta revisión, efectuada sobre los datos recogidos en once grupos de discusión, nos ha permitido identificar un conjunto de «tipos ideales» de participantes en los debates sobre el agua.

En lo que respecta a los «expertos», es factible establecer un primer elemento a resaltar. No solo se entiende por expertos a aquellos especialistas que se pronuncian desde la ciencia o la ingeniería y que cuentan con una acreditación académica que legitima su conocimiento. Por el contrario, se introduce una forma de experticia ciudadana que es altamente valorada y que, incluso, despierta una mayor confianza entre un sector de los entrevistados. Así, aunque se hace referencia a la importancia de la participación de científicos y técnicos en la controversia, su saber no es unánimemente entendido como el único autorizado para pronunciarse acerca del agua. Los «perjudicados» por planes y proyectos hidrológicos, sustentados en evaluaciones tecnocientíficas, también se consideran voces legítimas. Lo son en la medida en que poseen un conocimiento local, fundamentado en la experiencia continuada y cotidiana con las fuentes de agua, que puede resultar útil para la toma de decisiones políticas.

La importancia dada al saber de los "perjudicados» recuerda que la protesta ambiental en España ha estado caracterizada por el localismo (Jiménez, 2005), hecho que se ha manifestado claramente en las movilizaciones en contra del Plan Hidrológico Nacional de 2001 (Pont et al., 2002). Dicho fenómeno parece estar acompañado de una disminución de la legitimidad de los especialistas. Dadas sus relaciones percibidas con los poderes político-económicos y el carácter ideológico de su intervención ya identificado por otros autores (por ejemplo, Espluga, 2004, Lujan y Todt, 2007, o Carvalho, 2007), los entrevistados manifiestan una marcada desconfianza en la posibilidad de que el criterio de dichos expertos sea utilizado como único argumento para cerrar las controversias.

Ahora bien, la parcialidad de los posicionamientos no es exclusiva de los especialistas, es igualmente percibida en los «expertos contributivos no certificados». A ellos también se les reconoce como defensores de intereses particulares. Así pues, no es la falta de objetividad la que deslegitima la participación de científicos y técnicos en las controversias, sino su posición como representantes de las élites políticas y de los grupos de interés económicos que pueden lucrarse con los planes y los proyectos hidrológicos. Los gobernantes, los partidos políticos, el sector turístico y urbanístico, los regantes, las empresas hidroeléctricas o químicas, las depuradoras, las embotelladoras, son categorizados por los entrevistados, uno a uno, como actores «interesados» en tomar las decisiones de acuerdo con su propio beneficio. Su desatención a la afectación de intereses locales hace que los «afectados» sean percibidos como voces silenciadas que es necesario recuperar, aunque eso suponga poner en duda los resultados de las investigaciones científico-técnicas.

En resumen, la participación de los expertos en las controversias hidrológicas es frecuentemente percibida como una estrategia de poder. Su conoci- 
miento se concibe como una herramienta a través de la cual los «interesados» justifican las decisiones que desean tomar como un argumento que aplaca a los legos. Es visto como una forma de generar conformidad en la ciudadanía para disminuir su oposición a los proyectos y planes hidrológicos. Esto es especialmente notorio en lo que atañe a los llamados «beneficiados»: ciudadanos corrientes, usuarios domésticos, que obtienen algún tipo de ganancia secundaria de las decisiones tomadas por los «interesados», bajo la asesoría experta.

Los «beneficiados» son personas alejadas de las fuentes de agua en disputa que, sin embargo, recibirán parte de esos recursos, por ejemplo, en la forma de suministro domiciliario. Estos actores, igual que los especialistas, los «interesados» y los "perjudicados», tienen una visión parcial de las controversias. Sin embargo, la misma es el producto de una imposición o de una manipulación urdida mediante el uso de los medios de comunicación. Los «beneficiados» son vistos como ciudadanos que solo reciben información que apoya a las decisiones tomadas por los «interesados» y que, por esto mismo, pueden caracterizarse como "no concienciados». Para ellos, los debates sobre el agua no son un asunto controvertido, no suponen ningún tipo de incertidumbre.

Lo anteriormente comentado no elimina, en principio, la posibilidad de que existan ciudadanos a la vez «beneficiados» por las políticas hidrológicas y «concienciados» de su carácter controvertido. Sin embargo, esta posibilidad - teórica y empíricamente posible - no fue mencionada durante los grupos de discusión. Es frecuente, por el contrario, que las categorías de «no concienciados» $\mathrm{y}$ «beneficiados» se mezclen y se usen indistintamente para referirse al electorado valenciano y murciano. En oposición a este ciudadano que es visto como epistemológica y políticamente heterónomo (dependiente del criterio experto y de las decisiones de los «interesados»), surge un actor adicional que, junto con los "perjudicados», aparece como participante legítimo en las controversias sobre el agua: los ciudadanos "concienciados».

Los concienciados representan, en cierto sentido, un retorno al ideal de objetividad e imparcialidad. Son ciudadanos que han construido un criterio propio a partir de la revisión de diferentes fuentes de información disponible y que, al no ser ni beneficiarios ni perjudicados directos de las políticas hidrológicas, parecen no tener ningún interés en juego. Lo que les moviliza no es su propio provecho, sino una curiosidad intrínseca o una preocupación por los temas hidrológicos, en tanto que asuntos de actualidad y de relevancia nacional. Estos ciudadanos se encuentran físicamente alejados de las fuentes de agua que serán o son intervenidas, pero se encuentran próximos a los "perjudicados» porque no desatienden su punto de vista. También guardan alguna cercanía con los «beneficiados», debido a que su conocimiento es indirecto (se obtiene a través de los medios de comunicación), pero se distancian de ellos porque asumen una posición cautelosa frente a la información que reciben. Son sujetos autónomos, con "criterio» propio.

Todos los actores mencionados han sido tenidos en cuenta, por diferentes entrevistados, como participantes posibles en las controversias sobre el agua. Sin embargo, solo algunos de ellos cuentan con la legitimidad suficiente para 
intervenir de manera efectiva en la toma de decisiones. Los primeros son los especialistas, que, aunque están acompañados por la sospecha, producen un conocimiento que sigue siendo irrenunciable y necesario, por cuanto los asuntos hidrológicos tienen un alto componente científico-técnico. Los segundos son los ciudadanos representados por los "perjudicados» y los «concienciados», que complementan la experticia certificada con el saber local o que ayudan a realizar el examen desapegado de las diferentes formas de conocimiento en disputa. La aparición de estos últimos actores, en función de un proceso de categorización que funciona sobre dos ejes ("perjudicado»-«afectado», "concienciado»-«no concienciado»), hace necesario revisar los posibles efectos de la identidad social en las controversias sobre el agua en España.

El discurso en el que son los "perjudicados» los que pueden y deben decidir sobre un eventual trasvase, y que desconfían de los expertos pagados por las administraciones, de los informes que producen y de los medios que las distribuyen, así como de los ciudadanos «no concienciados» (especialmente si se trata de otras comunidades autónomas), tiene un marcado acento identitario. La aparición de la identidad colectiva, especialmente de corte territorial, en las controversias sobre el agua en España es coherente con investigaciones hechas sobre el ecologismo español (Jiménez, 2005), sobre el ecologismo catalán (Mora i Tico, 1999) y sobre la oposición al Plan Hidrológico Nacional y al trasvase del Ebro (Pont et al., 2002; Tàbara et al., 2004); así como con análisis de autores que han participado en los debates hidrológicos en los últimos años (Arrojo, 2006; Martínez Gil, 1997). Resulta, así, indispensable profundizar en este fenómeno para, así, crear estrategias que aprovechen el poder de movilización que puede llegar a tener la identidad, sin sacrificar la necesidad de diálogo entre los distintos actores que intervienen en los debates hidrológicos. Así mismo, significa preparar de antemano estrategias que disminuyan la posibilidad de que actores "de afuera", pero no necesariamente ilegítimos, se vean excluidos del debate. Obrar de esta manera implica reconocer, como afirman Tàbara et al. (2004), que no existe una única cultura del agua ni «una única moralidad o racionalidad a partir de las cuales poder prescribir unívocamente las mejores políticas ambientales» (p. 154).

Las sospechas que recaen sobre los científicos y los técnicos hacen necesaria, a su vez, una revisión del papel preponderante que se da a los procesos informativos y formativos en las controversias tecnocientíficas públicas. La percepción que se tiene del saber experto como saber parcializado y la concepción de los «beneficiados» como actores heterónomos debido a su aproximación acrítica a la información especializada, son indicios de que el llamado «modelo del déficit» es un viejo ideal puesto en duda por los ciudadanos catalanes. En su reemplazo, se consideran deseables estrategias activas de generación de conocimiento, ya sea mediante la experiencia directa (como en el caso de los "perjudicados») o mediante la construcción idiosincrásica de un juicio (como en los «concienciados»). Estas estrategias no han de ser solo respuestas coyunturales ante la necesidad de justificar un proyecto o un plan hidrológico, sino una actividad estructural y continua. Así, «educar desde la base a los peque- 
ños», y hacerlo desde una perspectiva contextualizada y crítica, parece fundamental para que la alfabetización tecnocientífica sea bien recibida y, a la vez, para que potencie realmente la participación ciudadana.

En definitiva, la constelación de actores rastreados en el discurso de los entrevistados dibuja un escenario donde la identidad colectiva y el conocimiento desempeñan un papel preponderante. En las páginas precedentes, la reflexión ha girado en torno a cómo la identidad de los participantes se delimita a partir de valoraciones hechas sobre el conocimiento que producen, obtienen, distribuyen, ocultan o construyen. Hemos visto que las categorías resultantes autorizan a determinados actores, y no a otros, a participar en las controversias sobre el agua. Dichas categorías pueden tener relaciones directas con la ubicación geográfica de los ciudadanos que intervienen en los debates. Adicionalmente, informan de saberes y de procesos de aprendizaje que son más apreciados que otros por los entrevistados. El diseño de espacios consultivos y participativos en materia hidrológica podrían tener en cuenta estas dimensiones psicosociales de la percepción general, a fin de potenciar la implicación del público en las controversias sobre el agua.

\section{Referencias bibliográficas}

AiBAR, E. (2002a). «La comprensión pública de la ciencia y la tecnología». En: AibAR, E. y QuinTANILLA, M.A. (eds.). Cultura tecnológica. Barcelona: Horsori / ICE de la Universidad de Barcelona, 127-146.

- (2002b). «La participación del público en las decisiones tecnológicas». En: AiBAR, E. y Quintanilla, M.A. (eds.). Cultura tecnológica. Barcelona: Horsori / ICE de la Universidad de Barcelona, 167-188.

Albiac, J.; Uche, J.; VAlero, A.; Serra, L.; Meyer, A. y TAPIA, J. (2003). «The economic unsustainability of the Spanish national hydrological plan». International Journal of Water Resources Development, 19 (3), 437-458.

ÁlvareZ, S.M. (2006). «La reforma de la legislación de aguas en España: Reflexiones sobre su necesidad y alcance». En: IBÁŃEZ, C. y PraTS, N. (eds.). Ciencia, técnica y ciudadaniá, claves para una gestión sostenible del agua. Zaragoza: Fundación Nueva Cultura del Agua, 457-470.

Arrojo, P. (ed.) (2001). El Plan Hidrológico Nacional, a debate. Bilbao: Bakeaz.

- (2006). El reto ético de la nueva cultura del agua: Funciones, valores y derechos en juego. Barcelona: Paidós.

BONÉ, A. (2003). «Reasons for the autonomous community of Aragon's position against the Ebro transfer as envisaged in the national hydrological plan». International Journal of Water Resources Development, 19 (3), 413-435.

CARVALHO, A. (2007). «Ideological cultures and media discourses on scientific knowledge: Re-reading news on climate change». Public Understanding of Science, 16 (2), 223-243.

Collins, H. y PINCH, T. (2002). Golem at large: What you should know about technology. Cambridge: Cambridge University Press.

EINSIEDEL, E.F.; JeLSøE, E. y BRECK, T. (2001). «Publics at the technology table: The consensus conference in Denmark, Canada, and Australia». Public Understanding of Science, 10 (1), 83-98. 
EMBID, A. (2003). "The transfer from the Ebro basin to the Mediterranean basins as a decision of the 2001 National Hydrological Plan: The main problems posed». International Journal of Water Resources Development, 19 (3), 399-411.

EsplugA, J. (2004). «Conflictes socioambientals i estudi sobre la percepció social del risc». Papers, 72, 145-162.

Goven, J. (2003). «Deploying the consensus conference in New Zeland: Democracy and de-problematization». Public Understanding of Science, 12 (4), 423-440.

GRAÑA, F. (2005). «¿Democratizar la democracia? Las nuevas formas de diálogo social». Boletin Cinterfor: Boletín Técnico Interamericano de Formación Profesional, 156, 125-148.

JimÉNEZ, M. (2005). El impacto político de los movimientos sociales: Un estudio de la protesta ambiental en España. Madrid: Centro de Investigaciones Sociológicas.

La Calle, A. (2006). "La Directiva Marco de Aguas en España: Una tarea por hacer». En: IbÁÑEZ, C. y PRATS, N. (eds.). Ciencia, técnica y ciudadanía, claves para una gestión sostenible del agua. Zaragoza: Fundación Nueva Cultura del Agua, 449456.

LEZAUn, J. y SONERYD, L. (2007). «Consulting citizens: Technologies of elicitation and the mobility of publics». Public Understanding of Science, 16 (3), 279-297.

LujAN, J.L. y TODT, O. (2007). «Precaution in public: The social perception of the role of science and values in policy making». Public Understanding of Science, 16, (1), 97-109.

MARTínez Gil, F.J. (1997). La nueva cultura del agua en España. Bilbao: Bakeaz.

Mora I Tico, P. (1999). "Una aproximació històrica a l'ecologisme català». Revista Catalana de Sociologia, 8, 65-94.

Mülberger, A.; Vilaró, M.; Tirado, F. y Domènech, M. (2007). «Historia, política y ciencia: El papel de los expertos en el debate sobre el agua en España». Centre d'Estudis d'Història de les Ciències y Grup d'Estudis Socials de la Ciència i la Tecnologia. URL. [http://www.recercat.net/bitstream/2072/4783/1/Recerca+historia+aigua+Espanya.pdf, consulta: 14 de febrer de 2008]

Pont, J.; Herrera, E.; MaXé, V. y Mena, E. (2002). La lluita per l'Ebre: El moviment social contra el Pla Hidrològic Nacional. Barcelona: Mediterrània.

RUBIO, M.J. y VARAS, J. (1997). El análisis de la realidad en la intervención social: Métodos y técnicas de investigación. Madrid: CSS.

SteneKes, N.; Colebatch, H.; Waite, D. y Ashbolt, N. (2006). «Risk and governance in water recycling: Public acceptance revisited». Science, Technology \& Human Values, 31 (2), 107-134.

STILGOE, J. (2007). "The (co-)production of public uncertainty: UK scientific advice on mobile phone health risks». Public Understanding of Science, 16 (1), 45-61.

STURGIS, P. y Allum, N. (2004). "Science in society: Re-evaluating the deficit model of public attitudes». Public Understanding of Science, 13 (1), 55-74.

TẢbara, J.D.; Costejà, M. y VAN WoERden, F. (2004). "Las culturas del agua en la prensa española: Los marcos culturales en la comunicación sobre el Plan Hidrológico Nacional». Papers, 73, 153-179.

Tytler, R.; DugGan, S. y GOTT, R. (2001). «Public participation in an environmental dispute: Implications for science education». Public Understanding of Science, 10 (4), 343-364. 